\section{Estrategia de uno en uno para mejorar la técnica correcta de higiene de manos}

\author{
Hernández-Orozco HG ${ }^{1}$, Castañeda-Narváez $\mathrm{JL}^{2}$, Lucas-Reséndiz $\mathrm{ME}^{3}$, Rosas- \\ Ruiz $\mathrm{A}^{4}$, Aparicio-Santiago $\mathrm{GL}^{5}$, Sandoval-Medina $\mathrm{MC}^{6}$
}

\begin{abstract}
Resumen
INTRODUCCIÓN: la higiene de las manos es considerada la estrategia más importante para prevenir infecciones asociadas con la atención de la salud. En nuestra institución, un estudio previo mostró que 93\% del personal del hospital dijo conocer la técnica de higiene de manos, pero las observaciones de vigilancia activa no reflejaban estos resultados.
\end{abstract}

OBJETIVO: mejorar la técnica de higiene de manos apropiada a través de una estrategia de "uno a uno".

MATERIAL Y MÉTODOS: estudio comparativo de antes y después, prospectivo, longitudinal, realizado entre 2012-2013, con personal de enfermería de cinco servicios. El estudio se realizó en tres etapas: la primer etapa consistió en observar la realización de higiene de manos reforzando verbalmente si era incorrecta; en la segunda etapa se pidió que se realizara la higiene de manos, si era incorrecta se pidió se repitiera realizándola al mismo tiempo con el observador; la tercera etapa fue la evaluación final de higiene de manos, después de al menos 15 días de la segunda etapa. Analizamos frecuencias de eventos correctos y realizamos comparaciones entre grupos por McNemar para evaluar si el efecto de la intervención se sostenía a pesar del tiempo; se evaluaron las mismas variables un año después del inicio del estudio.

RESULTADOS: la prueba de McNemar mostró diferencias estadísticamente significativas entre la realización de la higiene de manos correcta entre la etapa 1 en comparación con las siguientes 35 versus $85 \% ; p<0.001$.

CONCLUSIONES: se confirmó que la intervención dirigida logra mayor cooperación por parte del personal e incrementa la técnica correcta de higiene de manos a corto plazo.

PALABRAS CLAVE: higiene de manos, cumplimiento, técnica correcta.
${ }^{1}$ Maestra en Ciencias. Adscrita al Departamento de Infectología. Representante presidente de Comité de Infecciones Asociados a la Atención de la Salud. ${ }^{2}$ Médico Especialista C adscrito Depto. de Infectología.

${ }^{3}$ Maestra en salud Pública, Vocal de Enfermería CIAAS.

${ }^{4}$ Enfermera del INP, Vocal de Enfermería del CIAAS. ${ }^{5}$ Lic. en Enfermería, Vocal de Enfermería del CIAAS. ${ }^{6}$ Enfermera.

Instituto Nacional de Pediatría, Ciudad de México.

Recibido: 10 de junio del 2015

Aceptado: 14 de diciembre del 2016

Correspondencia

Hilda Guadalupe Hernández-Orozco

wuzhi1916@gmail.com

Este artículo debe citarse como

Hernández-Orozco HG, Castañeda-Narváez JL, Lucas-Reséndiz ME, Rosas-Ruiz A, Aparicio-Santiago GL, Sandoval-Medina MC. Estrategia de uno en uno para mejorar la técnica correcta de higiene de manos. Acta Pediatr Mex. 2017;38(5):299-307. 


\section{One-to-one training to increase correct hand hygiene technique.}

\author{
Hernández-Orozco HG ${ }^{1}$, Castañeda-Narváez $\mathrm{JL}^{2}$, Lucas-Reséndiz $\mathrm{ME}^{3}$, Rosas- \\ Ruiz $\mathrm{A}^{4}$, Aparicio-Santiago $\mathrm{GL}^{5}$, Sandoval-Medina $\mathrm{MC}^{6}$
}

\begin{abstract}
INTRODUCTION: Hand hygiene $(\mathrm{HH})$ is considered the most important strategy to prevent healthcare associated infections. At our institution, a previous survey showed that $93 \%$ of hospital staff said they knew the hand's hygiene technique. But active surveillance observations did not reflect these results.
\end{abstract}

OBJECTIVE: Improve appropriate hand hygiene technique through a strategy "one to one".

METHODS: Before and after study, conducted from 2012 to 2013 including the nursing staff. The study was done in three stages: $1 \mathrm{st}$ stage: we observed nurse performing $\mathrm{HH}$ and reinforced verbally if technique was incorrect. 2nd stage: we invited a nurse to perform a $\mathrm{HH}$, if technique was incorrect, we asked the nurse to do $\mathrm{HH}$ together with a trained observer. 3 rd stage: was performed with at least 15 days after 2nd stage and consisted in a final $\mathrm{HH}$ technique assessment. We analyzed and compared frequencies of correct events with ji2 and McNemar.

RESULTS: The McNemar test showed statistically significant difference between the frequencies of correct hand hygiene $(\mathrm{HH})$ at stage 1 compared with subsequent stages (35 versus $85 \%$; $\mathrm{P}<.001$ ).

CONCLUSIONS: We confirmed that led intervention increased cooperation by the staff and improved the correct technique of $\mathrm{HH}$ in the short term.

KEYWORDS: hand hygiene compliance; correct technique

\footnotetext{
${ }^{1}$ Maestra en Ciencias. Adscrita al Departamento de Infectología. Representante presidente de Comité de Infecciones Asociados a la Atención de la Salud.

${ }^{2}$ Médico Especialista C adscrito Depto. de Infectología.

${ }^{3}$ Maestra en salud Pública, Vocal de Enfermería CIAAS.

${ }^{4}$ Enfermera del INP, Vocal de Enfermería del CIAAS.

${ }^{5}$ Lic. en Enfermería, Vocal de Enfermería del CIAAS.

${ }^{6}$ Enfermera.
}

Instituto Nacional de Pediatría, Ciudad de México.

Correspondence

Hilda Guadalupe Hernández-Orozco

wuzhi1916@gmail.com

\section{INTRODUCCIÓN}

Las infecciones asociadas con la atención de la salud son una causa importante de morbilidad y de mortalidad. En los Estados Unidos cerca de dos millones de personas se infectan y 80,000 de ellas mueren anualmente por esta causa. La trasmisión cruzada más frecuente de agentes patógenos es a través de las manos contamina- das de trabajadores de la salud; esta afirmación es reforzada por el bajo cumplimiento de la higiene de manos reportada, generalmente por debajo de 50\%. ${ }^{1}$ Uno de los principales retos de la prevención de infecciones es lograr el cumplimiento de la realización de la higiene de manos. Algunas estrategias asociadas que determinaron el incremento de esta medida en los trabajadores de salud son: la educación en servicio y el uso de 
carteles y material didáctico, ${ }^{2}$ por lo cual en este estudio se realizó un práctica dirigida a personal de uno a uno con el objetivo de incrementar la correcta realización de la higiene de manos.

\section{MATERIAL Y MÉTODOS}

Estudio comparativo de antes y después, prospectivo, longitudinal realizado en el período 2012-2013, incluyó a todo el personal de enfermería que se encontraba en funciones en el momento del estudio, específicamente en los servicios del segundo piso de hospitalización que incluye: Unidad de Terapia Intensiva, Unidad de Terapia Intensiva Cardiovascular, Cardiología, Ortopedia, Clínica de Accesos Vasculares, Clínica de Estomas y Cirugía, reclutando una muestra por conveniencia de enfermeras y clasificando a los sujetos de acuerdo con su categoría en 4 grupos: enfermeras, enfermeras especialistas, jefe de enfermeras y supervisoras, en total 95 personas. Se excluyó al personal que no aceptó participar en el estudio y se eliminó a los sujetos donde no se pudo realizar la medición durante el seguimiento.

Se considera higiene de manos correcta cuando se aplica solución alcoholada a las manos y se siguen los seis pasos de la técnica de higiene de manos recomendada en la Guía de Higiene de Manos para personal de salud de la Organización Mundial de la Salud. El estudio se realizó en tres etapas en las cuales un personal de la unidad de vigilancia epidemiológica acudió en forma personalizada, es decir, interactuó con un personal de enfermería a la vez, para evaluar la correcta realización de la higiene de manos.

En la primera etapa se observó la realización de higiene de manos en el personal y, si no era correcta, se indicaba verbalmente al personal como debía realizarse la técnica comentándole se acudiría después para evaluar su técnica.

En la segunda etapa se acudió a evaluar la correcta higiene de manos y se pidió al personal realizarla; si había desviaciones se le invitaba a realizar la técnica correcta, al mismo tiempo que el observador lo practicaba y se le indicó se acudiría nuevamente a evaluar su técnica.

En una tercera etapa se hizo la última evaluación de la técnica de higiene de manos. Se dejaron pasar al menos 15 días entre cada etapa. Se utilizó un formato para recolección de datos epidemiológicos incluyendo turno, categoría y registro de los procedimientos correctos e incorrectos; en el Cuadro 1 se pueden observar los resultados

Cuadro 1. Frecuencia de realización adecuada de higiene de manos de acuerdo con las recomendaciones de la Organización Mundial de la Salud en personal de salud

\begin{tabular}{|l|c|c|c|c|}
\hline \multirow{2}{*}{ Etapas de estudio } & \multicolumn{4}{|c|}{ Clasificación por categoría de personal de salud* } \\
\cline { 2 - 5 } & $\begin{array}{c}\text { Enfermera } \\
(\mathbf{n = 4 5 )}\end{array}$ & $\begin{array}{c}\text { Enfermera especialista } \\
(\mathbf{n = 3 1 )}\end{array}$ & $\begin{array}{c}\text { Jefa de enfermeras } \\
(\mathbf{n = 9 )}\end{array}$ & $\begin{array}{c}\text { Supervisora de enfermería } \\
(\mathbf{n = 7})\end{array}$ \\
\cline { 2 - 5 } & $\mathbf{n}(\%)$ & $\mathbf{n}(\%)$ & $3(33.3)$ & $\mathbf{n}(\%)$ \\
\hline Primera etapa & $18(40.9 \%)$ & $10(33.3)$ & $8(83.3)$ & $5(11.1)$ \\
\hline Segunda etapa $(p \leq 0.001)$ & $37(81.8)$ & $21(66.7)$ & $8(83.3)$ & $6(77.9)$ \\
\hline Tercera etapa $(p \leq 0.001)$ & $41(90.9)$ & $21(66.7)$ & $7(75)$ & $5(66.7)$ \\
\hline $\begin{array}{l}\text { Al año de inicio del estudio } \\
(\mathbf{p} \leq 0.05)\end{array}$ & $33(74.4 \%)$ & $31(100)$ & &
\end{tabular}

*Se utiliza la prueba de McNemar para determinar cambio de los grupos. 
de la correcta higiene de manos. Los sujetos que no completaron las tres etapas fueron eliminados del análisis. Se solicitó consentimiento de todos los participantes del estudio.

Análisis estadístico descriptivo: frecuencias y comparación entre grupos y etapas de observación por prueba de McNemar.

\section{RESULTADOS}

Un total de 92 sujetos completó todas las fases del estudio y se realizó la medición por servicio y por categoría. La observación inicial mostró un porcentaje bajo de la realización correcta de la técnica de higiene de manos de acuerdo con lo recomendado por la Organización Mundial de la Salud, siendo el menor en supervisoras de enfermería con $11 \%$ y el mayor se presentó en enfermeras con $41 \%$ (Cuadro 1). En la primera etapa participaron 94 sujetos, pero se eliminaron 2 ya que no realizaron las siguientes etapas. En forma global el promedio de realización de la técnica de higiene de manos en forma correcta fue de $35 \%$, por lo que se tuvo que reforzar verbalmente la técnica en $77 \%$ de los participantes.

En la segunda etapa se logró un incremento en la correcta técnica de higiene de manos en todos los grupos con resultados de $67 \%$ el menor a $83 \%$ el mayor (Cuadro 1). Considerando todos los grupos el cumplimiento promedio en la segunda etapa fue de $80 \%$ y en la tercera etapa se logró 85\% (Figura 1); en el grupo de supervisoras y enfermeras se obtuvo una mejora de 77 y $91 \%$, respectivamente, con la basal; sin embargo, en los otros grupos se mantuvo el porcentaje de realización de higiene de manos observado en la segunda etapa. La prueba de McNemar mostró diferencia significativa entre la medición de los grupos antes y después de la estrategia $(p \leq 0.001)$. No se observaron diferencias en cuanto al turno de personal en las distintas categorías $(p>0.05)$. Se realizó

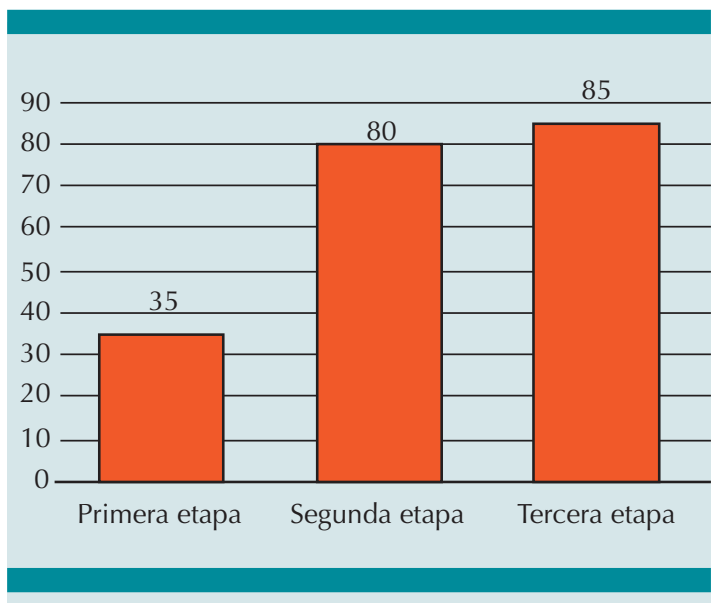

Figura 1. Avance porcentual en la realización de la correcta higiene de manos en las diferentes etapas.

una medición al año de iniciado el estudio y se observó una disminución del efecto de la intervención en los grupos de enfermería, jefes de enfermera y supervisoras, no así en el grupo de enfermeras especialistas. Cabe mencionar que la técnica correcta se realizó en más de $67 \%$ por arriba de la medición basal (Cuadro 1) lo que correspondió a un incremento de $37 \%$ con una $p$ significativa $(p<0.01)$. El análisis por servicio encontró una realización correcta de higiene de manos basal de 21 a 60\%, excepto en Clínica de Accesos Vasculares y Clínica de Estomas en donde se presentó uniformemente de $100 \%$ en la basal y las etapas de seguimiento.

El servicio de unidad de cuidados intensivos pediátricos y unidad de cuidados intensivos cardiovasculares aumentaron a 90\% la realización correcta de higiene de manos, mientras que los servicios de cirugía y ortopedia alcanzaron un $89 \%$ y en el departamento de cardiología un $78 \%$, por lo que podemos deducir que en estos servicios la intervención fue positiva. Sin embargo, de la segunda a la tercera etapa la estrategia no mostró ningún cambio en unidad de cuidados intensivos cardiovasculares que había alcanzado un $90 \%$ de realización correcta de la técnica de 
higiene de manos desde la segunda etapa. Así mismo, en la Unidad de accesos vasculares y clínica de estomas se corroboró que el personal conocía la técnica y la realizaba seguramente porque sus actividades incluyen dentro de su procedimiento a la higiene de manos como un punto fundamental para el adecuado desarrollo del mantenimiento del catéter y de la realización del procedimiento de curación de una herida (Figura 2).

\section{DISCUSIÓN}

La guía para la realización de higiene de manos de la Organización Mundial de la Salud refiere que la técnica más económica, práctica y de fácil acceso para prevenir infecciones asociadas a la atención de la salud es realizar higiene de manos con agua y jabón o soluciones con base de alcohol, con la ventaja de que el uso de esta última permite realizar una técnica de higiene de manos en menor tiempo y con la misma efectividad; por ello, actualmente se recomienda promover la higiene de manos realizada con soluciones alcoholadas. En la guía se describe la técnica de realización de higiene de manos mediante seis pasos que cubren toda la superficie de las manos, siguiendo secuencialmente las superficies de palmas, dorso, espacios interdigitales, nudillos, dedo pulgar y uñas; se considera que si es con agua y jabón, el tiempo para realizarlo es de 40 a 60 segundos y si se utiliza solución alcoholada el tiempo sólo es de 20 a 30 segundos con una reducción de $50 \%$ en su realización. ${ }^{3}$ La técnica observada en el estudio fue la descrita en la guía de la Organización Mundial de la Salud (Figura 3).

Suárez y sus colaboradores refieren que entre 50 y $60 \%$ del personal de salud son enfermeras, de las cuales solo $43 \%$ realiza correctamente la técnica de higiene de manos. ${ }^{4}$ Este personal es el que ocupa mayor tiempo en la atención directa del paciente, por lo que cómo realizan su trabajo

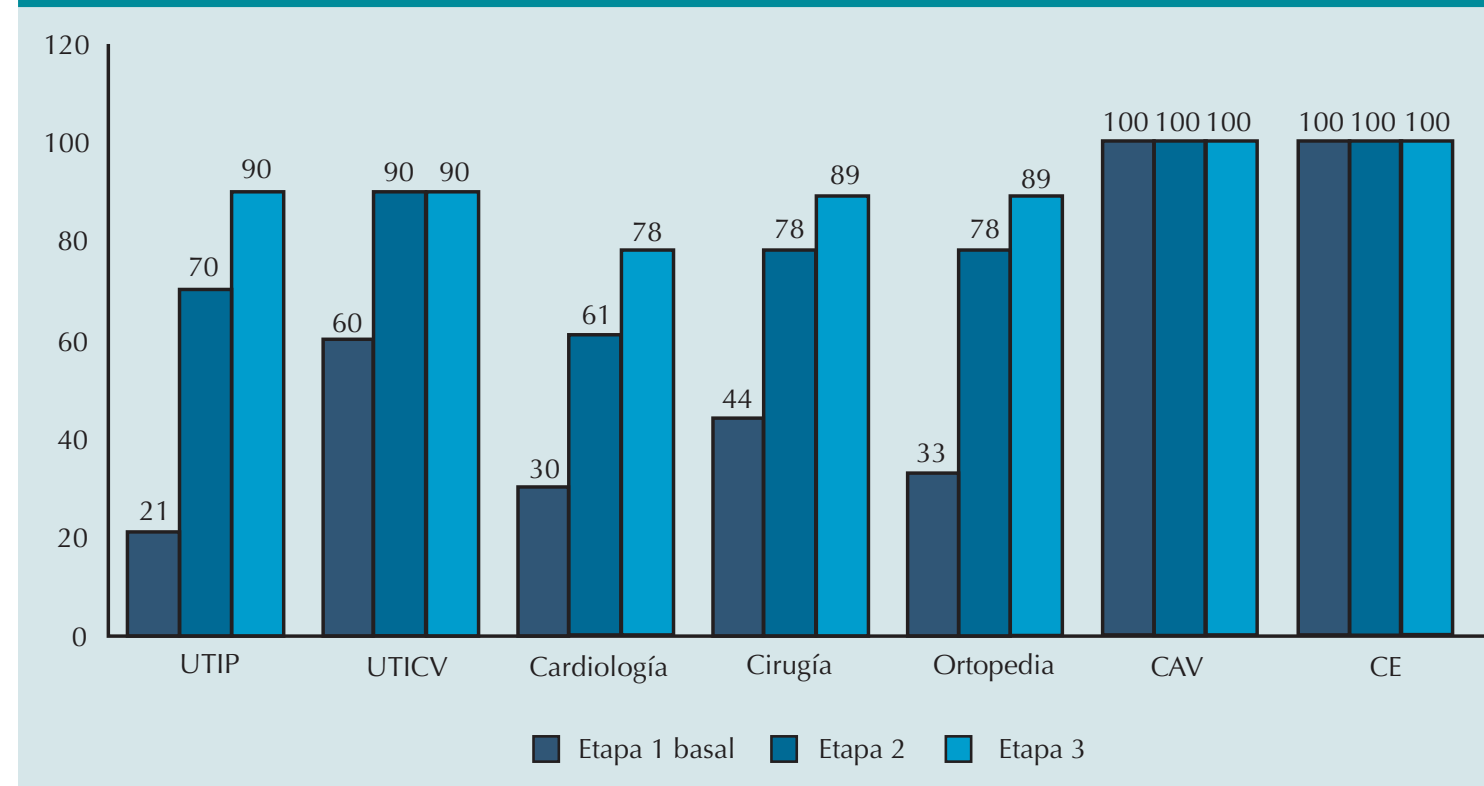

CAV: clínica de accesos vasculares; CE: clínica de estomas; UTICV: unidad de terapia intensiva cardiovascular; UTIP: unidad de terapia intensiva.

Figura 2. Avance porcentual en la realización de la correcta higiene de manos, por servicio, en las diferentes etapas. 


\section{¿Cómo desinfectarse las manos?}

¡Desinféctese las manos por higiene! Lávese las manos solo cuando estén visiblemente sucias

(1) Duración de todo el procedimiento: 20-30 segundos

12
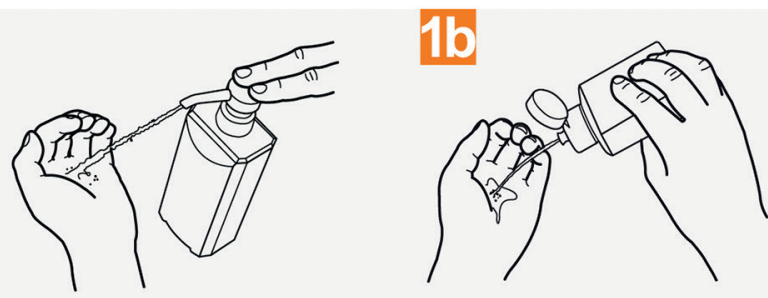

Deposite en la palma de la mano una dosis de producto suficiente para cubrir todas las superficies;

3

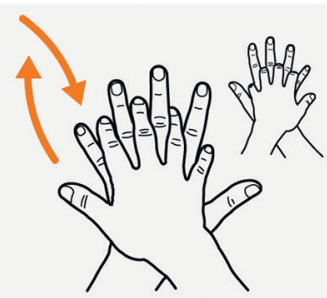

Frótese la palma de la mano derecha contra el dorso de la mano izquierda entrelazando los dedos y viceversa;

\section{6}

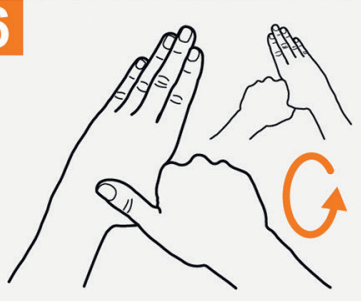

Frótese con un movimiento de rotación el pulgar izquierdo, atrapándolo con la palma de la mano derecha y viceversa;

\section{4}

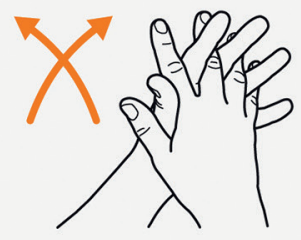

Frótese las palmas de las manos entre sí, con los dedos entrelazados;
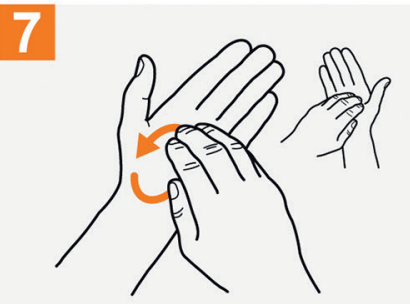

Frótese la punta de los dedos de la mano derecha contra la palma de la mano izquierda, haciendo un movimiento de rotación y viceversa;
2

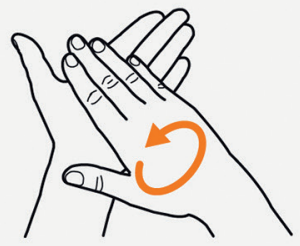

Frótese las palmas de las manos entre si;

5

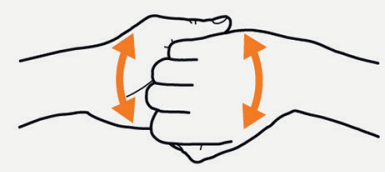

Frótese el dorso de los dedos de una mano con la palma de la mano opuesta, agarrándose los dedos;

8

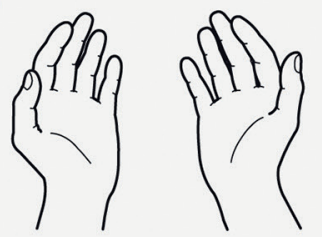

Una vez secas, sus manos son seguras.

Figura 3. Técnica de higiene de manos. 
diario y los procedimientos, son vitales para romper la cadena de trasmisión de la infección. Este cumplimiento es mayor a lo encontrado en nuestro estudio en la etapa basal. Al igual que en el estudio de Suarez y su equipo se encontró una mayor adherencia a la realización de higiene de manos en licenciados de enfermería (57\%). ${ }^{4}$ En nuestro estudio los resultados en la tercera etapa de evaluación reportó que $91 \%$ de la realización de higiene de manos correcta fue en este grupo, siendo el más alto, seguido por las jefes de enfermeras. En este grupo las supervisoras, por su actividad de asesoría, debían tener un mayor conocimiento de esta medida; sin embargo, fue el grupo que obtuvo menor porcentaje de realización correcta de higiene de manos, aumentando a $78 \%$ en la etapa 3 , por lo que es fundamental que se capacite a este personal en forma adecuada, ya que es el personal normativo al resto del personal.

Al final de nuestro estudio se observó mayor realización de higiene de manos en las unidades de terapia intensiva pediátrica y de terapia intensiva cardiovascular (90\%) al igual que lo observado por Suárez, quien mencionan un mejor cumplimiento de higiene de manos en unidades de cuidados intensivos de $50 \% .^{4}$ Esto es positivo ya que en estos servicios existe mayor riesgo de infección por las condiciones del paciente y el uso de más procedimientos invasivos para diagnóstico y tratamiento. Fue satisfactorio observar en el estudio que la Clínica de accesos vasculares y la clínica de estomas realizó en $100 \%$ de las oportunidades observadas una adecuada higiene de manos que indica una excelente capacitación, compromiso y responsabilidad. Pitet y sus colaboradores ${ }^{5}$ mencionan que un programa de promoción de higiene de manos con énfasis en el uso de soluciones alcoholadas incrementó la realización de higiene de manos de 48 a 66\%, incremento significativo en enfermeras y asistentes de enfermería; sin embargo, en médicos el cumplimiento de higiene de manos permanece bajo. En dicho estudio aumenta el consumo de soluciones alcoholadas de 3.5 a 15.4 por 1000 días y disminuyen las infecciones asociadas a la atención de la salud de 16.9 a $9.9 \%$ y de S. aureus meticilino-resistente de 2.16 a 0.93 por 1000 días paciente; ${ }^{5}$ en nuestro estudio la realización de higiene de manos fue con solución alcoholada y sólo se observó personal de enfermería. Según Jiménez y sus colegas ${ }^{6}$ la enfermería es una profesión de entrega y dedicación que da importancia a la atención del paciente en forma integral; sin embargo, al olvidar la higiene de manos en el proceso de atención, todo el esfuerzo será inútil, ya que repercute en la presencia de infecciones asociadas a la atención de la salud, mayor comorbilidad asociada e incluso la muerte del paciente. ${ }^{6}$

Kilpatrick y su grupo ${ }^{7}$ mencionan que se ha simplificado la realización de higiene de manos en el hospital al aplicar el concepto de los cinco momentos, ya que la higiene de manos debe realizarse en el área de atención del paciente Ilamada zona o área del paciente y en cinco indicaciones básicas, momento 1 al entrar en la zona del paciente; momento 2 antes de tener contacto con el paciente; momento 3 realizar tareas donde existe el riesgo de contaminarse con sangre o secreciones del paciente; momento 4 después de atender al paciente; momento 5 cinco al salir de la zona del paciente. La Figura 4 muestra los 5 momentos de la higiene de manos. También los autores comentan la importancia de buscar nuevas estrategias para lograr la higiene de manos como el uso de videos donde se demuestren las situaciones en donde debe llevarse a cabo esta medida y la promoción a través de las redes sociales de la técnica de higiene de manos y la monitorización de ésta. ${ }^{7}$

Sería interesante conocer si esta estrategia puede ser aplicada a otros grupos de personal de salud, principalmente al grupo médico, debido a que se presenta un bajo apego al cumplimiento 


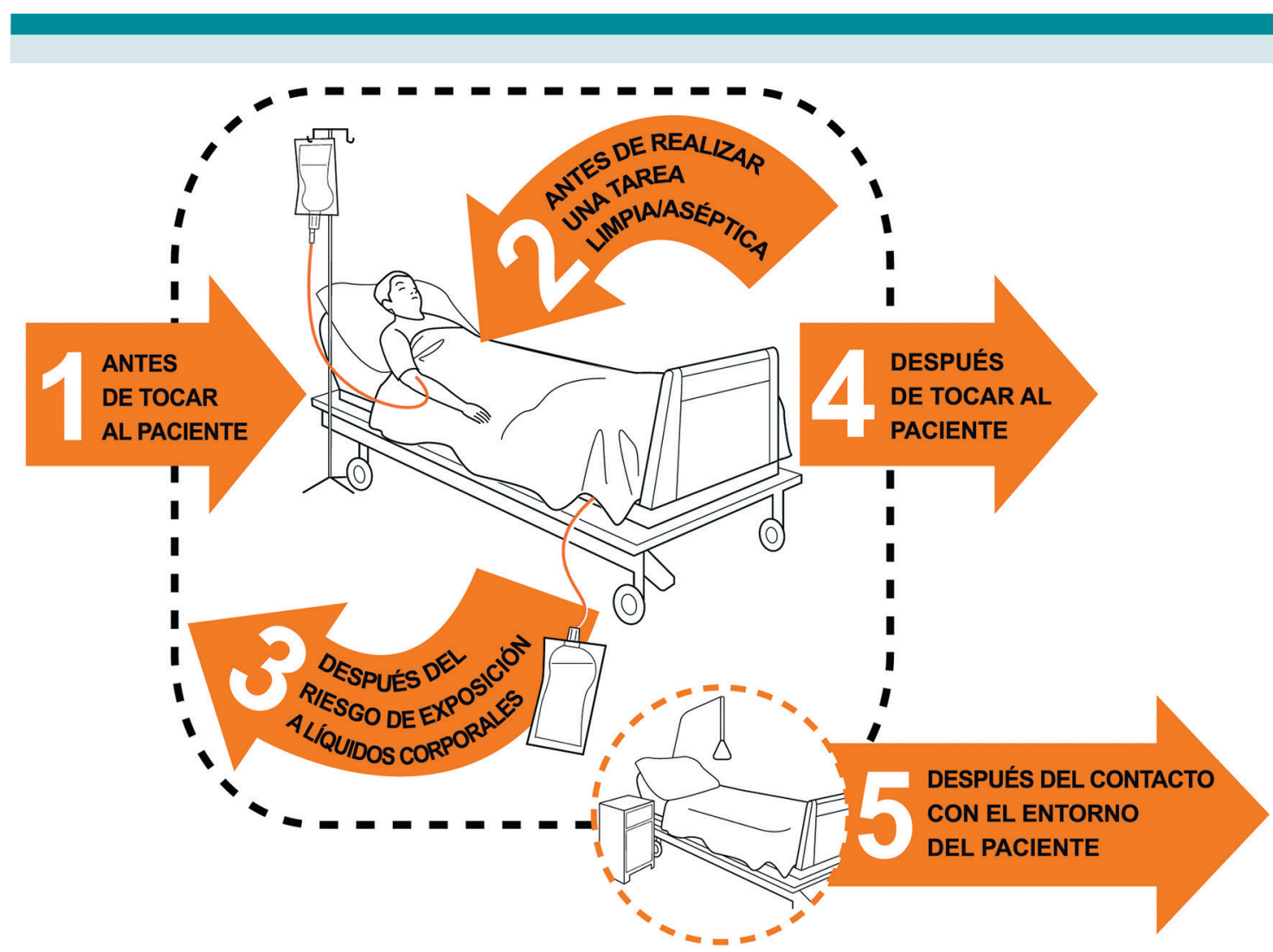

Figura 4. Los 5 momentos de realización de higiene de manos, Organización Mundial de la Salud.

consecuencia de considerar esta medida como una técnica de rutina que fácilmente se olvida. ${ }^{7}$ Boscart y sus colaboradores, en un estudio cualitativo, encontró que la higiene de manos fue en gran medida un proceso rutinario y automático para las enfermeras. ${ }^{8}$

Hay evidencia de que es compatible la influencia de colegas en el cumplimiento de la higiene de manos, nuestro estudio demostró en la etapa 2 mejoría en el apego al ser supervisados. Los modelos de conducta se sabe que juegan un papel central en el cambio de conducta de los médicos en general, y para mejorar el cumplimiento de la higiene de manos específicamente.
Curiosamente, se encuentra una diferencia entre especialidades con respecto a la influencia de otros miembros del equipo en las prácticas de higiene de las manos, lo que indica que la importancia de las influencias sociales puede variar según la especialidad. Los cirujanos declararon que no eran influidos por otros miembros del equipo en su práctica de la higiene de manos, tal vez debido a que los cirujanos deben trabajar más independientemente que los médicos que tienden a trabajar más en equipo. ${ }^{9}$

Las limitaciones del sistema en cuanto a recursos son una barrera importante para que el médico realice higiene de manos, principalmente a 
facilitar el acceso en el sitio de atención del paciente. Las altas cargas de trabajo también se surgieren como una limitación.

Una limitante de la estrategia es que al dar una atención personalizada al personal de salud, por parte de una de los miembros de la unidad de vigilancia epidemiológica, puede disminuirse el tiempo para realizar sus otras actividades, lo que conllevaría evaluar la necesidad de un mayor número de miembros en la unidad de vigilancia epidemiológica si quisiera implementarse esta estrategia o buscar participantes fuera de ella como la estrategia de acompañante con líderes voluntarios en los servicios.

\section{CONCLUSIÓN}

Este estudio demuestra que una intervención dirigida de persona a persona logra una mayor cooperación por el personal y el incremento de la realización de la técnica adecuada de higiene de manos a corto y mediano plazos. La importancia de continuar con la capacitación y reforzamiento de esta medida buscando nuevas estrategias, como sugieren Kilpatrick y sus colegas, ${ }^{7}$ se ve reflejado en lo observado al año de aplicación de esta estrategia en donde se encontró una disminución leve de la realización correcta de higiene de manos en personal de enfermería.

Este estudio permite corroborar que es un acierto que en nuestra institución se mantenga un programa permanente de higiene de manos Ilamado "Burbuja de la Vida"; ¿ por qué lo llamamos así?, simplemente porque de manera virtual una burbuja significa protección al paciente, en este caso contra los microorganismos que se pueden trasmitir a través de las manos, pero también en sentido inverso al proteger a otros, al contener dentro de la burbuja la flora que coloniza la piel del paciente y entonces no habría trasmisión al exterior.
Los resultados del estudio son la razón por la que capacitamos sobre higiene de manos frecuentemente al personal. Muchos refieren que ya lo saben, sin embargo, como observamos en la medición al año del estudio, la higiene de manos disminuyó en parte del personal evaluado, lo que genera que siempre tengamos que capacitar y buscar estrategias que permitan recordar a todos lo importante que es la realización de este procedimiento, esperando que el cumplimiento global sea idealmente de $100 \%$ y al menos mayor a $70 \mathrm{u} 80 \%$.

La limitación del estudio es que no se describió que las observaciones no eran cegadas al personal, ya que ellos conocían se efectuaría la evaluación del uso de la técnica correcta de higiene de manos en las segunda y tercera etapas.

\section{REFERENCIAS}

1. Institute Healthcare Improvement (IHI). How to guide improvement hand hygiene. Disponible en: http://www. shea-online.org/Assets/files/IHI_Hand_Hygiene.pdf Acceso: $1 / 01 / 2015$

2. Pitet D. Improving adherence to hand hygiene practice: a multidisciplinary approach. Emerg Infect Dis. 2001;7(2):234-240.

3. WHO Guidelines on Hand Hygiene in Health Care: a Summary 2009. Disponible en: http://www.who.int/ gpsc/5may/tools/who_guidelines-handhygiene_summary. pdf. Acceso 1/10/2012

4. Suárez ST, Guanche HG, Cañal NA, Maldonado GC, Benítez MM. Adherencia a la realización de lavado de manos por el personal de enfermería en un hospital clínico quirúrgico de la Habana. Hig Salud Amb. 2013;13(1):940-45.

5. Pitet $D$, Hugonnet $S$, Harpagh $S$, Mourouga P, Sauvan V, Touveneau S, Parreger TV. Effectiveness of a hospital wide programmed to improve compliance with hand hygiene. Lancet. 2000;356(9238):1307-12.

6. Jiménez ALS, Prado VV. El impacto actual del lavado de manos. Med Nat. 2008;2(2):123-9.

7. Kilpatrick C, Hosie L, and Storr J. Hand hygiene when and how should it be done? Nursing Times. 2013;109(38):16-8.

8. Boscart M, Fernie GR, Lee JH Jaglal SB. Using psychological theory to inform methods to optimize the implementation of a hand hygiene intervention Veronique. Implementation Science. 2012;7(77):1-12.

9. Squires JE, Linklater S, Girmshaw JM, Graham DI, Sulivan K, Bruce N, Gartke K, Karovitch A et al. Understanding Practice: Factors That Influence Physician Hand Hygiene Compliance. Infect Control Hosp Epidemiol. 2014;35(12):1511-1520. 